\title{
Dipole Interactions with Random Anisotropy in a Frozen Ferrofluid
}

\author{
Weili Luo, (1) Sidney R. Nagel, (1) T. F. Rosenbaum, ${ }^{(1)}$ and R. E. Rosensweig ${ }^{(2)}$ \\ (1) The James Franck Institute and Department of Physics, The University of Chicago, Chicago, Illinois 60637 \\ ${ }^{(2)}$ Corporate Research Science Laboratories, Exxon Research and Engineering Company, Annandale, New Jersey 08801
} (Received 21 June 1991)

\begin{abstract}
Glassy behavior (including hysteresis, irreversibility, a peak in the zero-field-cooled magnetization, and nonexponential relaxation) is observed in a quenched ferrofluid system consisting of 50- $\AA$ magnetite particles. An Arrott plot, $M^{2}$ vs $H / M$, shows clear features of random anisotropy similar to what is found in amorphous ferromagnets. We discuss the glassy behavior in terms of both the random anisotropy and the dipole interactions, and we contrast the unusual response of our system with canonical spin glasses.
\end{abstract}

PACS numbers: $75.50 . \mathrm{Lk}, 75.50 . \mathrm{Mm}$

Magnetically disordered systems have been intensively studied in recent years [1]. In particular, spin glasses, with competing interactions, and random-field magnets, with random effective magnetic fields at the local sites, have been the focus of much attention. Very little work has been done on systems with dipole interactions largely because millikelvin temperatures are required in order to achieve ordering [2]. Recently, disordered systems with random magnetic anisotropy [3] have been investigated both theoretically and experimentally. Such systems involve magnetic moments with easy magnetization axes, due to local crystal fields, which are different from site to site. The theory [4] for such systems was originally developed in order to explain the unusual behavior of amorphous ferromagnets. Systems with random anisotropy plus competing interactions remain essentially unexplored. In this paper we report studies on a system which has both dipole-dipole interactions and local random anisotropy.

The experiments were performed on a ferrofluid, a solution of magnetic particles suspended in a nonmagnetic solvent [5] as shown schematically in the illustration in Fig. 1. For our sample the magnetic material is magnetite, $\mathrm{Fe}_{3} \mathrm{O}_{4}$, with a mean particle diameter of $50 \AA$ and a standard deviation of $\pm 16 \AA$. Each particle is a ferrimagnetic microcrystal. In order to avoid clustering, each particle is coated with a $\sim 20$ - $\AA$-thick nonmagnetic surfactant layer. The Curie temperature for bulk magnetite is $\approx 850 \mathrm{~K}$ [6], which is much higher than the freezing temperature of $210 \mathrm{~K}$ of the solvent, a hydrocarbon oil. For our particle size there is a single domain within the particle [5], and to a very good approximation we can take each whole grain as a rigid moment at low temperature. The average moment of each particle $\mu$ is about $3000 \mu_{B}$, where $\mu_{B}$ is the Bohr magneton [7]. Earlier magnetization measurements of ferrofluid samples [8] involved much larger particles so that the moment was constrained to point along the easy axis of the particle. Thus, each dipole became immobilized at the freezing temperature of the solvent. In our system, by contrast, the dipoles can change their orientation below the freezing point of the solvent and the ground state is determined by the magnetic interactions between the dipoles.
The moments interact with one another through the magnetic-dipole interaction: $J_{i j}=\mu_{i} \mu_{j}\left(1-3 \cos ^{2} \theta_{i j}\right) / r^{3}$. The large $\mu_{i}$ moves the characteristic interactions from the millikelvin to the kelvin temperature range. This interaction can be either ferromagnetic or antiferromagnetic depending on the relative orientation of each dipole. This suggests that a disordered dipolar system may behave as a spin glass [2,9]. Inside each particle the crystal field creates easy axes for the magnetic moment of the grain. Electron microscopy has indicated that the particles in our sample are basically spherical so that shape anisotropy is unimportant. For temperatures slightly below $100 \mathrm{~K}$, the crystal structure of magnetite changes from cubic to monoclinic and the system develops a uniaxial easy axis [10]. As the solvent freezes, each easy axis is frozen in a random orientation so that the only relaxation process is spin rotation relative to the particle. The spins experience a random anisotropy due to the frozen easy axes.

Our sample, sealed inside a small quartz tube with epoxy, was placed in a SQUID magnetometer and cooled in zero field from room temperature to a temperature below the freezing temperature of the solvent. During a

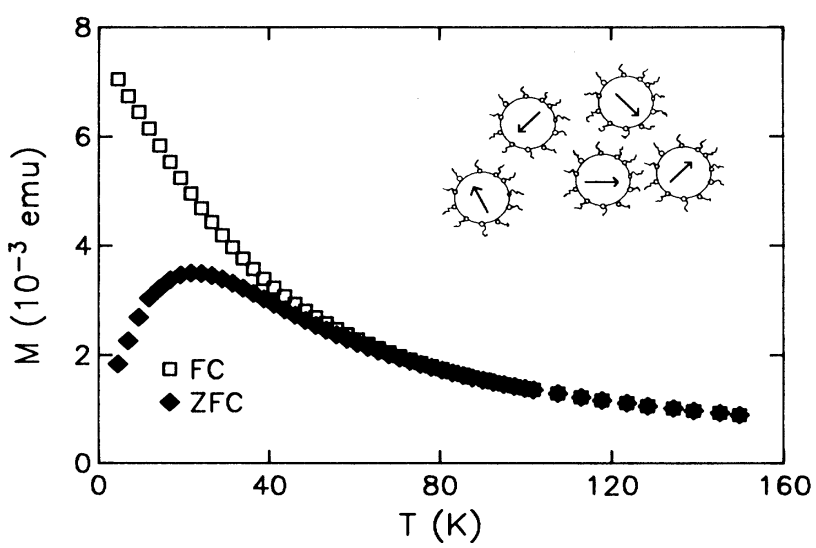

FIG. 1. Field-cooled (FC) and zero-field-cooled (ZFC) magnetization vs temperature at $H=10 \mathrm{G}$. The illustration shows randomly oriented magnetite particles of median diameter $50 \AA$ and a surfactant layer of $\sim 20 \AA$. 
measurement the magnetic-field variation is less than $0.05 \%$. The fluid was examined by electron microscopy and no agglomeration was found.

The main part of Fig. 1 shows the magnetization $M$ versus temperature $T$ in a field $H=10 \mathrm{G}$. The lower curve, marked ZFC (for zero-field cooled), was obtained by first cooling the system in zero magnetic field from 150 to $4.5 \mathrm{~K}$. The magnetic field $H$ is then applied and the magnetization is measured while the temperature is increased. The upper curve, marked FC (for field cooled), is obtained in a similar manner except that the sample is cooled in the measuring field. The two curves are the same for temperatures above about $80 \mathrm{~K}$, but depart from one another at lower temperatures. The ZFC curve peaks at $T_{\max }=21 \mathrm{~K}$ and the FC curve continues to increase with decreasing temperature. Both the fact that the two curves depart from one another at a temperature much higher than $T_{\text {max }}$ and the fact that the field-cooled magnetization continues to increase without saturation below $T_{\max }$ distinguish this system from what is observed in canonical spin-glass systems. We presume that the distribution of particle size produces a broad peak in the ZFC curve instead of a sharp cusp as in a spin glass. Nanometer particles in an insulating medium also show a peak in the ZFC curve and an FC curve which continues to increase on lowering the temperature [11]. However, in those cases the peak in the ZFC curve is associated with the superparamagnetic blocking temperature which depends on the time scale of the measurement. The time dependence of the peak in our ZFC curve is considerably slower than what would be expected from superparamagnetic blocking and appears to saturate at long times. This indicates that even in the limit of infinite waiting times, the peak would occur at a finite temperature. In addition, as we explain below, the evidence suggests that our peak is due to interactions between the grains.

The peak in the ZFC curve is field dependent as shown in Fig. 2, where $T_{\max }$ is plotted against $H$. To determine whether $T_{\max }$ is a collective effect or simply the blocking temperature of a superparamagnet, the dependence of $T_{\max }$ on dipole concentration was explored. Samples with different volume fractions of magnetite, $\varepsilon \equiv V_{m} / V$, were obtained by changing the amount of solvent. Thus, the distribution of particle sizes should be the same in all samples. Figure 2 also shows the $H$ dependence of $T_{\max }$ for the different concentrations $\varepsilon=0.002,0.02$, and 0.04 . For low fields $T_{\max }$ shifts nonlinearly to higher values with increasing concentration. All the curves merge at about a few hundred gauss; for fields larger than this value, $T_{\max }$ decreases with increasing field for all the samples. The largest $T_{\max }$ is $26 \mathrm{~K}$, found in the most concentrated sample. In order to calculate the magnetic energy associated with this temperature we set $\mu H_{0}$ $=k_{B} T_{\max }$ and obtain $H_{0}=150 \mathrm{G}$. This suggests that for fields larger than $150 \mathrm{G}$ the magnetic energy dominates

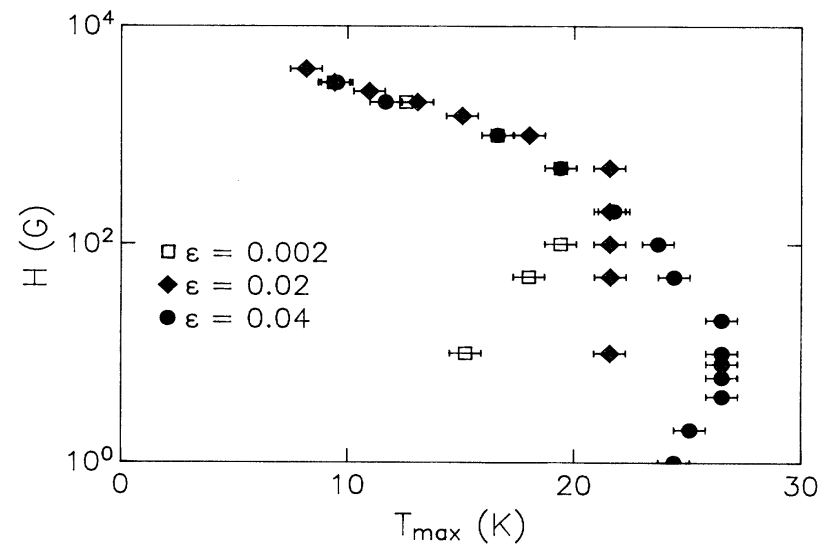

FIG. 2. $H$ vs $T_{\max }$, the peak position in the ZFC curve, for three volume fractions $\varepsilon$ of magnetite. At low fields the interactions dominate, with all curves merging at large $H$.

the interaction energy. In this regime the differences between the concentrations should be diminished. The field where the curves merge approximately coincides with $\boldsymbol{H}_{0}$ and lends credence to the idea that for magnetic fields less than a few hundred gauss the interaction energy dominates the behavior.

It is worth comparing our results for $T_{\max }(H)$ with work on the canonical spin glasses $\mathrm{Ag}: \mathrm{Mn}$ and $\mathrm{Cu}: \mathrm{Mn}$. In those materials, $T_{\max }$ is roughly independent of $H$ for small fields [12]. In contrast, for our samples we find an unusual increase in $T_{\max }$ with increasing field at small $H$. Here, the application of an external field apparently increases the barriers to spin reorientation. In the spin glasses, the field dependence of the irreversible temperature $T_{\text {irr }}$, below which the ZFC and FC curves start to differ, is consistent with the de Almeida-Thouless line [12,13]. For the ferrofluid, the irreversibility starts very gradually far above $T_{\max }$ and the irreversible temperature $T_{\text {irr }}$ is difficult to specify.

In order to determine the anisotropy strength, we have measured hysteresis curves. Figure 3 shows the results at $4.5 \mathrm{~K}$ for the $\varepsilon=0.02$ mixture. We obtain the anisotropy constant $K$ from the following relations: $K=\frac{1}{8}$ times the area of the hysteresis cycle [14] and the anisotropy for magnetite, $K_{m}=K / \varepsilon \approx 2.3 \times 10^{4} \mathrm{ergs} / \mathrm{cm}^{3}$. The temperature corresponding to the anisotropy energy $D$ is $D / k_{B}$ $=K_{m} V / k_{B} \approx 10 \mathrm{~K}$, where $k_{B}$ is the Boltzmann constant. We are in the range $D / J \approx 1$ (we have used $J=2 \mu^{2} / r^{3}$ $\approx 5 \mathrm{~K}$ to estimate the exchange interaction between two spins). The remanence is $M_{r}=0.21 M_{0}$, where $M_{0}$ is the saturation magnetization. For an assembly of noninteracting, randomly oriented single-domain particles one expects [15] $M_{r}=0.5 M_{0}$. Hadjipanayis and co-workers [16] pointed out that when there are ferromagnetic interactions in the system, $M_{r}>0.5 M_{0}$, but if there are antiferromagnetic interactions, $M_{r}<0.5 M_{0}$. The reduced $M_{r}$ is further evidence for interactions which can lead to frustration. 


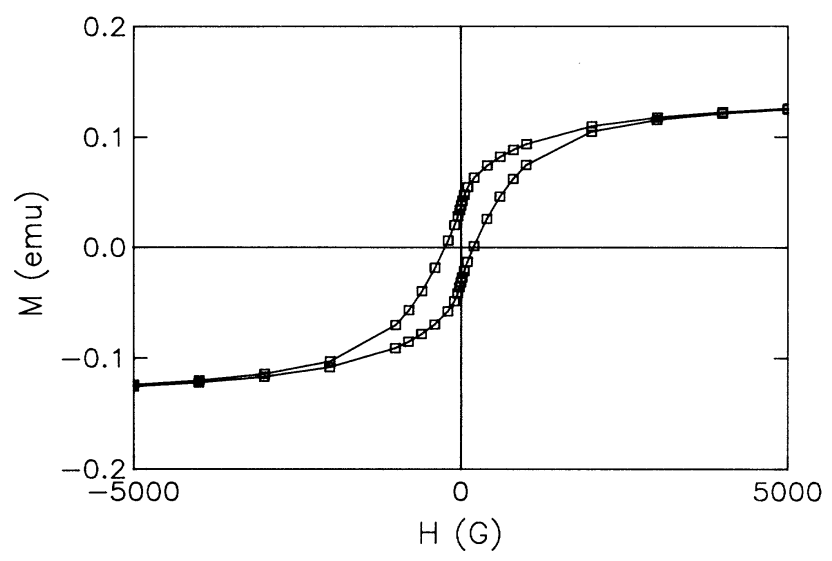

FIG. 3. Hysteresis curve at $T=4.5 \mathrm{~K}$ for a sample with $\varepsilon=0.02$ obtained by field cooling.

Aharony and Pytte [17] showed within a mean-field approach that for a system with ferromagnetic interactions the local uniaxial anisotropy will destroy the longrange order. In an Arrott plot, where $M^{2}$ is plotted against $H / M$, the curvature should change at a specific temperature. This temperature was found to be related to the onset of the glassy behavior. Although the Aharony-Pytte theory is only valid for weak anisotropy, a similar change of curvature was also found in amorphous ferromagnetic materials with both weak and strong anisotropy [3,18]. In all these materials the interaction among magnetic moments is basically ferromagnetic. No predictions exist for random anisotropic systems with competing interactions. We show in Fig. 4 an Arrott plot for our ferrofluid sample. A change of the curvature is obvious at $T \geq T_{\max }$. A change of curvature in an Arrott plot is not unique to amorphous ferromagnets; it also occurs in a dipolar system with random anisotropy.

Magnetic relaxation results are presented in Fig. 5. The sample was cooled in a field of $H=100 \mathrm{G}$ from 150 to $15 \mathrm{~K}$, at which point the field is cut to zero and the time decay of the thermoremanent magnetization (TRM) was recorded by the SQUID magnetometer. The data are well fitted by a logarithmic form. However, we note that similar $\ln t$ fits were observed over short time intervals in spin glasses, but were later found to be inadequate to describe the data over a large range [1]. From $10^{2}$ to $10^{4} \mathrm{sec}$ the remanence decreases by only $20 \%$, indicating that the relaxation time is much longer than a few hours. In the inset in Fig. 5 we show the logarithmic slope of the decay versus $T$. It peaks at $T<T_{\max }$, as was also observed in studies of the Au:Fe spin glass [19], amorphous ferromagnets [16], and in the vortex state of high- $T_{c}$ superconductors [20].

The logarithmic time dependence and the nonmonotonic temperature dependence for $M(t)$ presented in Fig. 5 are consistent with the predictions of a recent model [21] for nonexponential relaxation based on dipole-dipole interactions. We note that nonexponential relaxation may

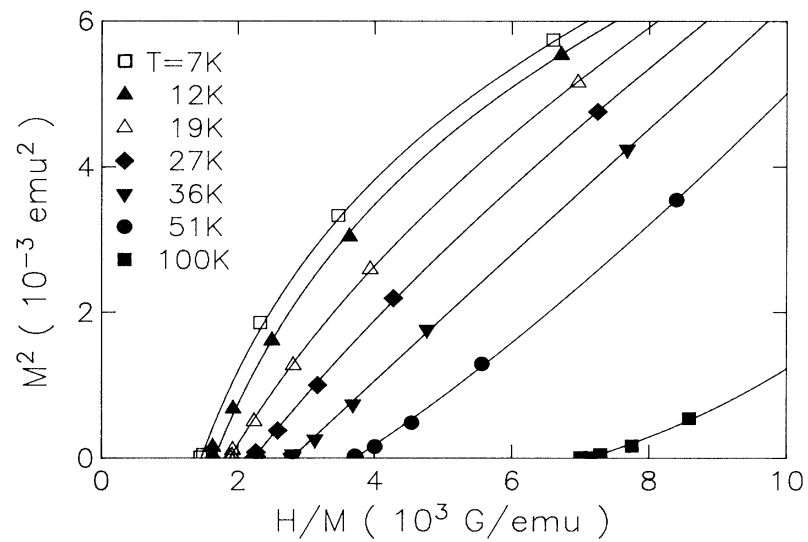

FIG. 4. Arrott plot, $M^{2}$ vs $H / M$ for a sample with $\varepsilon=0.02$, with a clear curvature change at $T \sim 36 \mathrm{~K}$, characteristic of a system with random anisotropy. Solid lines are guides to the eye.

also be associated with a distribution of relaxation times in the system, arising either (i) from competing interactions among magnetic moments and disorder giving rise to a multivalley structure in the free-energy surface or (ii) from the distribution of the anisotropy energies. When a magnetic field is turned off, each moment attempts to return to the easy-axis direction defined by the anisotropy energy. The Néel relaxation time for this process is $\tau_{N}=\tau_{0} \exp \left(K_{m} V / k_{B} T\right)$, where $\tau_{0}=10^{-9} \mathrm{sec}$, and $K_{m}$ and $V$ are the anisotropy constant and volume for each particle, respectively [5]. The distribution of particle sizes produces a distribution of relaxation times. Using the value of $K_{m}$ obtained from the hysteresis loop in Fig. 3, we find $\tau_{N} \approx 1.0 \times 10^{-8} \mathrm{sec}$ for a particle with the average diameter occurring in our sample $(50 \AA)$ at $T=4.5 \mathrm{~K}$. A relaxation time longer than $10^{4} \mathrm{sec}$ would

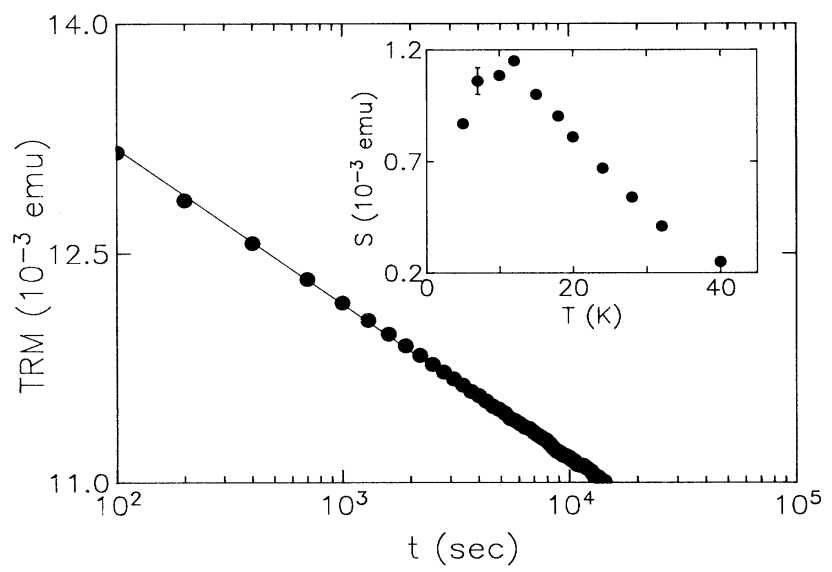

FIG. 5. Time decay of the thermoremanent magnetization for $H=100 \mathrm{G}, T=15 \mathrm{~K}$. Within our time window, the decay can be fitted by a logarithmic form (solid line). Inset: The slope of the $\ln t$ fit, $S$, vs temperature. The peak in $S$ is seen in many types of glassy systems. 
require a particle with a diameter larger than $190 \AA$ at $T=20 \mathrm{~K}$. Even if such large particles are present, their contribution to the magnetization is negligible. We conclude from the time dependence that the collective interaction [21] plays an important role in the glassy behavior.

Given that both the dipole interaction and the RKKY interaction [1] in metallic spin glasses are long range, one might expect that they should behave in a similar manner. Our experimental results indicate many common features between the dipole system and canonical spin glasses, i.e., irreversibility, hysteresis, a peak in the ZFC magnetization, and slow nonexponential relaxation. Hysteresis in our system is more reminiscent of what is found in $\mathrm{Au}: \mathrm{Fe}$ spin glasses than what is seen in $\mathrm{Cu}: \mathrm{Mn}$ spin glass. The difference between the two spin glasses is the presence of significant spin-orbit scattering in $\mathrm{Au}: \mathrm{Fe}$, which causes local anisotropy [14]. The hysteresis loop of $\mathrm{Au}: \mathrm{Fe}$ has a smooth curve rather than an abrupt jump seen in the Cu:Mn spin glass [14]. This feature is another confirmation of random local anisotropy in our system.

There are two major differences between our system and a standard spin glass. First, we find a curvature change in the Arrott plot. von Molnar and co-workers [18] compared random-anisotropy and spin-glass properties in several amorphous rare-earth alloys. They found that for amorphous rare-earth ferromagnets the curvature changes at a specific temperature in the Arrott plot, and that this temperature is associated with the peak temperature in the zero-field-cooled magnetization. However, for the spin glass $\mathrm{Gd}_{0.37} \mathrm{Al}_{0.63}$, there is no curvature change in the Arrott plot from a temperature above $T_{g}$ to temperatures below $T_{g}$, where $T_{g}$ is the spin-glass transition temperature. The results shown in Fig. 4 indicate that our system behaves in this respect more like a random-anisotropy system than a normal spin glass. A second difference is that the FC curve of our quenched ferrofluid sample continues to increase with decreasing temperature, while for a spin glass the corresponding curve flattens off below the freezing temperature. Whether this increase in the FC magnetization below $T_{\max }$, representing continuing degrees of freedom for the spins below the nominal ordering, is unique to dipolar systems with random anisotropy or a more general phenomenon is not understood.

We thank J. Popplewell and S. W. Charles for providing the samples used in this work. We are grateful to $\mathrm{T}$. Witten, K. Levin, J. Marko, and J. Rudnick for stimulating discussions. The work at the University of Chicago was supported by NSF-MRL Grant No. DMR88-19860.

[1] K. Binder and A. P. Young, Rev. Mod. Phys. 58, 801 (1986).

[2] D. H. Reich et al., Phys. Rev. B 42, 4631 (1990).

[3] For a review, see K. Moorjani and J. M. D. Coey, Magnetic Glasses (Elsevier, Amsterdam, 1984), and refer- ences therein.

[4] R. Harris, M. Plischke, and M. J. Zuckermann, Phys. Rev. Lett. 31, 160 (1973).

[5] R. Rosensweig, Ferrohydrodynamics (Cambridge Univ. Press, Cambridge, 1985).

[6] O. Steinsvoll and T. Riste, J. Magn. Magn. Mater. 14, 187 (1979); P. Auric et al., J. Non-Cryst. Solids 50, 97 (1982); Y. Shimizu, J. Geomagn. Geoelect. 11, 125 (1960).

[7] The average moment was determined by (i) using the value of the moment in bulk magnetite and multiplying by the number of $\mathrm{Fe}_{3} \mathrm{O}_{4}$ in a particle and (ii) measuring the magnetization at room temperature and determining the relative volume occupied by ferrofluid. The two estimates agreed within $10 \%$ and indicate that the moment per $\mathrm{Fe}_{3} \mathrm{O}_{4}$ is approximately the bulk value. Our samples are an order of magnitude larger than the Fe [W. A. de Heer, P. Milani, and A. Châtelain, Phys. Rev. Lett. 65, 488 (1990)] and Co [J. P. Bucher, D. C. Douglass, and L. A. Bloomfield, Phys. Rev. Lett. 66, 3052 (1991)] clusters in which a significant decrease in the magnetic moment was observed [S. N. Khanna and S. Linderoth, Phys. Rev. Lett. 67, 742 (1991)].

[8] A. A. Minakov et al., Izv. Akad. Nauk SSSR, Ser. Fiz. 51, 1062 (1987); A. F. Pshenichnicov and M. I. Shliomis, ibid. 51, 1067 (1987).

[9] A. Aharony, Phys. Rev. B 13, 2092 (1976); A. Aharony and M. J. Stephen, J. Phys. C 14, 1665 (1981).

[10] For bulk magnetite, the structural transition temperature, the Verway temperature $T_{V} \approx 120 \mathrm{~K}$, decreases with decreasing particle size. See K. Abe, Y. Miyamoto, and S. Chikazumi, J. Phys. Soc. Jpn. 41, 1894 (1976); M. Matsui, S. Todo, and S. Chikazumi, J. Phys. Soc. Jpn. 43, 47 (1977); S. Morup and H. Topsoe, J. Magn. Magn. Mater. 31-34, 953 (1983).

[11] S. H. Liou and C. L. Chien, J. Appl. Phys. 63, 4240 (1988); A. Gavrin and C. L. Chien, J. Appl. Phys. 67, 938 (1990).

[12] R. V. Chamberlin et al., Phys. Rev. B 25, 6720 (1982); R. V. Chamberlin, Ph.D. thesis, UCLA, 1984 (unpublished); G. G. Kenning, D. Chu, and R. Orbach, Phys. Rev. Lett. 66, 2923 (1991).

[13] J. R. L. de Almeida and D. J. Thouless, J. Phys. A 11, 983 (1978).

[14] J. J. Prejean and M. J. Joliclerc, J. Phys. (Paris) 41, 427 (1980).

[15] E. C. Stoner and E. P. Wohlfarth, Philos. Trans. Roy. Soc. London A 240, 599 (1948).

[16] G. Hadjipanayis, D. J. Sellmyer, and B. Brandt, Phys. Rev. B 23, 3349 (1981); G. Hadjipanayis and D. J. Sellmyer, Phys. Rev. B 23, 3355 (1981).

[17] A. Aharony and E. Pytte, Phys. Rev. Lett. 45, 1583 (1980).

[18] S. von Molnar et al., J. Appl. Phys. 53, 2350 (1982); S. von Molnar, T. R. McGuire, and R. J. Gambino, ibid. 53, 7666 (1982).

[19] C. N. Guy, J. Phys. F 8, 1309 (1978).

[20] A. Hamzic, L. Fruchter, and I. A. Campbell, Nature (London) 345, 515 (1990); C. Rossel, Y. Maeno, and I. Morgenstern, Phys. Rev. Lett. 62, 681 (1989).

[21] D. K. Lottis, R. M. White, and E. D. Dahlberg, Phys. Rev. Lett. 67, 362 (1991). 